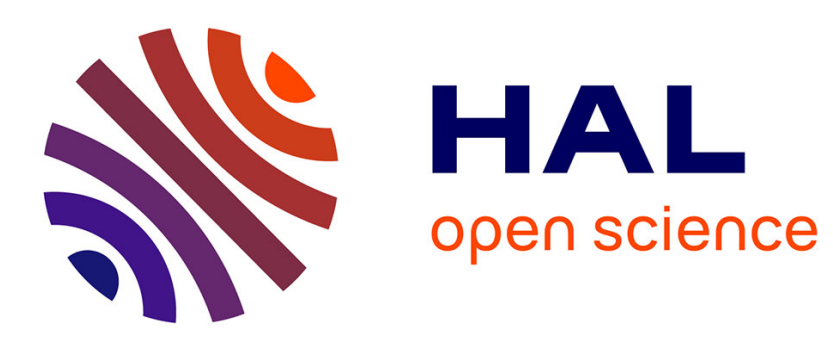

\title{
Natural deduction calculi and sequent calculi for counterfactual logics
}

Francesca Poggiolesi

\section{To cite this version:}

Francesca Poggiolesi. Natural deduction calculi and sequent calculi for counterfactual logics. Studia Logica, 2016, 104 (5), pp.1003-1036. 10.1007/s11225-016-9662-3 . hal-01271556

\section{HAL Id: hal-01271556 \\ https://hal.science/hal-01271556}

Submitted on 21 Jun 2018

HAL is a multi-disciplinary open access archive for the deposit and dissemination of scientific research documents, whether they are published or not. The documents may come from teaching and research institutions in France or abroad, or from public or private research centers.
L'archive ouverte pluridisciplinaire HAL, est destinée au dépôt et à la diffusion de documents scientifiques de niveau recherche, publiés ou non, émanant des établissements d'enseignement et de recherche français ou étrangers, des laboratoires publics ou privés. 


\section{Francesca Poggiolesi}

Tenured researcher,

Université Paris 1 Panthéon-Sorbonne, CNRS, ENS,

UMR 8590 IHPST -

Institut d'Histoire et de Philosophie des Sciences et des Techniques,

poggiolesi@gmail.com 


\title{
Natural deduction calculi and sequent calculi for counterfactual logics
}

\begin{abstract}
In this paper we present labelled sequent calculi and labelled natural deduction calculi for the counterfactual logics CK $+\{$ ID, MP $\}$. As for the sequent calculi we prove, in a semantic manner, that the cut-rule is admissible. As for the natural deduction calculi we prove, in a purely syntactic way, the normalization theorem. Finally, we demonstrate that both calculi are sound and complete with respect to Nute semantics [12] and that the natural deduction calculi can be effectively transformed into the sequent calculi.
\end{abstract}

\section{Introduction}

Conditional sentences are sentences of the form "if A, then C" and they are a central topic in logic and philosophy of logic. Standardly, one can distinguish between two types of conditional: indicative conditionals, i.e. conditionals of the form "if John strikes the match, it will light", and counterfactual (or strict) conditionals, i.e. conditionals of the form "if John had struck the match, it would have lit". The main difference between the two types of conditional consists in the fact that, while in counterfactual conditionals the antecedent is supposed to be false, in indicative conditionals it can either be true or false. In this paper we will only focus on counterfactual conditionals, see $[4,11]$.

Though often criticized, the standard interpretation of indicative conditionals is the material one: a conditional sentence is true if, and only if, either the antecedent is false or the consequent is true. If one tries to apply the material interpretation to counterfactual conditionals, since their antecedent is taken to be false, one gets the conclusion that all counterfactuals are trivially true and this is of course inadequate. For this reason other semantics interpretations of counterfactuals have been proposed.

Amongst them (e.g. $[7,8]$ ) the possible worlds semantics interpretation stands out $[10,12,20]$. The intuitive idea of such semantical approach is the following. Consider the sentence "if John had struck the match, it would have lit." In the actual world John did not strike the match. Consider then the possible world(s), which is (are) the most similar to the actual one, where John actually stroke the match. If in that world(s), the match had lit, than the counterfactual is true in the actual world. In other terms, since counterfactual sentences talk about unrealized possibilities, in order to check whether they are true in the actual world, one needs to go to the possible world(s) where they actually took place and explore their consequences.

There are several ways in which such an intuitive idea has been rendered. Amongst them, the most famous ones are Stalnaker's approach [20], Lewis's 
approach [10] and Nute's approach [12]. The main differences amongst them are the following: Stalnaker's approach involves a set of possible words plus an accessibility relation and a selection function; Lewis's approach is based on the so-called system of spheres; finally, Nute's approach only comprehends a set of possible worlds and a selection function. In this paper we will only take into account Nute semantics and certain systems that such semantics characterize, namely the systems $\mathrm{CK}+\{\mathrm{ID}, \mathrm{MP}\}$.

From a proof-theoretical point of view, little work has been done for the systems $\mathrm{CK}+\{\mathrm{ID}, \mathrm{MP}\}$. On the sequent calculi side, ${ }^{1}$ the only works we are aware of are those of Olivetti, Pozzato and Schwind [14] and Alenda, Olivetti, Pozzato [1]. More precisely, in Olivetti, Pozzato and Schwind [14] sequent calculi for counterfactual logics based on Nute semantics are constructed by means of labelled formulas of the form $x: A$ and $x R^{A} y$; in Alenda, Olivetti, Pozzato [1] sequent calculi for the same logics are constructed by means of deep-inferences or tree-hypersequents, see $[16,17]$. On the natural deduction side, there is, as far as we know, no result to mention. ${ }^{2}$

Given this situation the main contributions of the paper are the following. First of all, we introduce labelled sequent calculi for the systems CK $+\{$ ID, $\mathrm{MP}$, that serve as a base to develop related labelled natural deduction calculi for the same systems. These natural deduction calculi fill (at least partially) the gap in the literature previously mentioned. As for the sequent calculi, we propose a semantic proof of completeness, from which it follows, as corollary, the admissibility of the cut-rule. The completeness proof is original and, given the presence of Nute's normality condition, not trivial. As for the natural deduction calculi we adapt the proof of syntactic cut-elimination elaborated by [1] for treehypersequents to the case of derivations in natural deduction calculi. We end the paper by showing that we can effectively transform natural deduction calculi for $\mathrm{CK}+\{\mathrm{ID}, \mathrm{MP}\}$ into sequent calculi for $\mathrm{CK}+\{\mathrm{ID}, \mathrm{MP}\}$.

The paper is organized in the following way. Section 2 will serve to introduce in detail Nute semantics, the systems $C K+\{\mathrm{ID}, \mathrm{MP}\}$ and the general idea of the labels that we want to use to build sequent calculi and natural deduction calculi for the systems $\mathrm{CK}+\{\mathrm{ID}, \mathrm{MP}\}$. In Section 3 we will introduce the calculi $\mathrm{GCK}^{*}$ and we will explore some of their properties. Section 4 will be dedicated to the semantic proof of soundness and completeness of $\mathrm{GCK}^{*}$ with respect to Nute semantics. In Section 5 we will introduce the natural deduction calculi $\mathrm{NCK}^{*}$ and we will prove some of their properties; in Section 6 we will show that in these calculi derivations normalize. Section 7 will be dedicated to the proof of the adequateness of natural deduction calculi $\mathrm{NCK}^{*}$ via a comparison with sequent calculi $\mathrm{GCK}^{*}$; finally, Section 8 will serve to draw some conclusions and to depict some lines of future research.

\footnotetext{
${ }^{1}$ A labelled tableaux system based on Nute semantics has been proposed by [2]. Sequent calculi for conditional logics characterized by Stalnaker semantics and Lewis semantics have been proposed by, amongst others, [5, 6, 13, 15].

${ }^{2}$ Natural deduction calculi for conditional logics characterized by Stalnaker semantics have been proposed by [21]; see also [9].
} 


\section{Nute semantics, the corresponding Hilbert sys- tems and the general idea for their proof the- ory}

We start this section by introducing the language $\mathcal{L}^{>}$that extends the language of classical logic by means of the operator $>$. The operator $>$ denotes counterfactual conditional.

Definition 2.1. The language $\mathcal{L}^{>}$is composed of:

- propositional atoms (PA): p, q, r ..

- the connectives: $\neg, \wedge, \rightarrow$ and $>$

- the parentheses $($,

The connectives $\vee$ and $\leftrightarrow$ are defined classically as usual. The symbol $\perp$ is defined as $A \wedge \neg A$ for any formula $A$.

Definition 2.2. The set of formulas FA of the language $\mathcal{L}^{>}$is given by the rule:

$$
p|\neg A| A \wedge B|A \rightarrow B| A>B
$$

We now introduce Nute models. Nute models are basically Kripke models where the accessibility relation is substituted by a selection function.

Definition 2.3. A Nute-model $\mathfrak{M}$ is a triple $(W, f, v)$, based on the frame $\mathfrak{F}=$ $(W, f)$, where:

- $W$ is non empty set of possible worlds,

- $f$ is the so-called selection function:

$$
f: W \times F A \rightarrow 2^{W}
$$

- $v$ is the evaluation function:

$$
v: W \times P A \rightarrow\{1,0\}
$$

Definition 2.4. The satisfability relation is defined in the following inductive way:

- $i \models_{\mathfrak{M}} p$ iff $v(i, p)=1$,

- $i \models_{\mathfrak{M}} \neg A$ iff $i \not \nvdash_{\mathfrak{M}} A$,

- $i \models_{\mathfrak{M}} A \wedge B$ iff $i \models_{\mathfrak{M}} A$ and $i \models_{\mathfrak{M}} B$

- $i \models_{\mathfrak{M}} A \rightarrow B$ iff if $i \models_{\mathfrak{M}} A$, then $i \models_{\mathfrak{M}} B$ 
Figure 1: Extensions of the Hilbert system CK

\begin{tabular}{|c|c|c|}
\hline Name & Axiom & Semantic Frame Property \\
\hline ID & $A>A$ & $\forall i \forall j\left(j \in f(i, A) \rightarrow j \models_{\mathfrak{M}} A\right)$ \\
MP & $(A>B) \rightarrow(A \rightarrow B)$ & $\forall i\left(i \models_{\mathfrak{M}} A \rightarrow i \in f(i, A)\right)$ \\
CEM & $(A>B) \vee(A>\neg B)$ & $|f(i, A)| \leqslant 1$ \\
& $((A>B) \wedge(B>A)) \rightarrow((A>C) \rightarrow(B>C))$ & $\forall i \forall j\left(j \in f(i, B) \rightarrow j \models_{\mathfrak{M}} A\right)$ implies \\
CSO & $\forall i \forall j\left(j \in f(i, A) \rightarrow j \models_{\mathfrak{M}} B\right)$ and \\
& \multicolumn{2}{|c}{} \\
\hline
\end{tabular}

- $i \models_{\mathfrak{M}} A>B$ iff $\forall j\left(j \in f(i, A) \rightarrow j \models_{\mathfrak{M}} B\right)$

The first four items of the satisfability relation are standard; we then dwell on the last one, which concerns counterfactual conditionals. A world $i$ satisfies a conditional of the form $A>B$ if, and only if, in all worlds $j$, which are the most similar to $i$ with respect to $A, B$ is satisfied. The satisfability relation satisfies the following condition, which is usually called normality condition:

if $A \leftrightarrow C$ is valid in the class of Nute frames, then $f(i, A)=f(i, C)$

The semantics above characterizes the basic conditional system CK, which is composed of:

- all tautologies of classical propositional logic,

- Modus Ponens,

- (RCEA)

$$
\frac{A \leftrightarrow B}{(A>C) \leftrightarrow(B>C)}
$$

- $(\mathrm{RCK})$

$$
\frac{\left(A_{1} \wedge \cdots \wedge A_{n}\right) \rightarrow B}{\left(C>A_{1} \wedge \cdots \wedge C>A_{n}\right) \rightarrow(C>B)}
$$

Other systems are obtained by adding to the system CK the axioms which are shown in Figure 1. In this paper we will only deal with the following three extensions of the system CK: $\mathrm{CK}+\mathrm{ID}, \mathrm{CK}+\mathrm{MP}$ and $\mathrm{CK}+\mathrm{ID}+\mathrm{MP}$. With the notation $\mathrm{CK}^{*}$ we will refer to the Hilbert systems $\mathrm{CK}, \mathrm{CK}+\mathrm{ID}, \mathrm{CK}+\mathrm{MP}$ and $\mathrm{CK}+\mathrm{ID}+\mathrm{MP}$. 
As it is clear from Definition 2.3, Nute semantics is a very simple semantics only composed by a set of possible worlds and a selection function. Another equivalent way of understanding Nute semantics is to see it as composed of worlds that can either be considered as worlds tout court or they can be viewed as worlds selected by a selection function. This last distinction lead us to the idea of creating a similar distinction amongst formulas to be used in the proof theory for the systems characterized by Nute semantics. We can indeed think of dealing not only with formulas of the language $\mathcal{L}^{>}$, i.e. formulas tout court, but also with labelled formulas, i.e. formulas of the type

$$
A_{j}: B
$$

which stands for: let $j$ be one of the worlds selected by the selection function $f(i, A)$, then $B$ is true in that world $j$. Another example of a labelled formula is the following one:

$$
C_{z} ; A_{j}: B
$$

let $z$ be one of the worlds selected by the selection function $f(i, C)$ and $j$ one of the worlds selected by the selection function $f(z, A)$, then B is true in that world $j$. Yet a final example is the following one:

$$
C_{z} ; A_{j} ; D_{k}: B
$$

let $z$ be one of the worlds selected by the selection function $f(i, C), j$ one of the worlds selected by the selection function $f(z, A)$, and $k$ one of the worlds selected by the selection function $f(j, D)$, then B is true in that world $k$.

The general idea here is to work with formulas of the language $\mathcal{L}^{>}$which are linked by a colon to sequences of formulas of $\mathcal{L}^{>}$indexed with the letters $i, j, k \ldots$ The formulas of the sequence together with their indexes denote the worlds selected by the selection function of a Nute model. When the sequence is empty, the labelled formula becomes a formula belonging to FA (see Definition 2.2) and corresponds to a world of a Nute model which has not been selected by a selection function. Let us express this idea in a more precise way.

Definition 2.5. Let $G, H, \ldots$ be sequences of formulas of $\mathcal{L}^{>}$separated by a semicolon and indexed in the following way:

$$
A_{j}^{1} ; B_{z}^{2} ; \ldots ; E_{t}^{n}
$$

where the upper index indicates the position of the formula in the sequence, while the lower index is just a letter. $G, H, \ldots$ could also be empty sequences.

Definition 2.6. From now on we will only work with labelled formulas. A labelled formula is an object of the following form:

$$
G: A
$$

where $G$ is a sequence of the type described above and $A$ is a formula of $\mathcal{L}^{>}$. 
By means of labelled formulas we are going to construct sequent calculi and natural deduction calculi for the systems $\mathrm{CK}^{*}$.

\section{Sequent Calculi}

We will use this section for introducing labelled sequent calculi for the systems $\mathrm{CK}^{*}$. We will then prove that in these calculi several structural rules are (heightpreserving) admissible and that logical rules are height-preserving invertible.

Definition 3.1. Let $M$ and $N$ be finite multisets of labelled formulas, then a sequent is an object of the following form: $M \Rightarrow N$.

Definition 3.2. We extend the satisfability relation (see Definition 2.4) to labelled formulas and sequents in the following way:

$$
\begin{aligned}
& i \models_{\mathfrak{M}} B_{j}^{1} ; G: A \text { iff } \forall j\left(j \in f(i, B) \rightarrow j \models_{\mathfrak{M}} G: A\right) \\
& i \models_{\mathfrak{M}} M \Rightarrow N \text { iff } i \models_{\mathfrak{M}} \bigwedge M \rightarrow \bigvee N
\end{aligned}
$$

We are going to use the following syntactic notations which will prove useful for formulating the rules of our calculi.

- Given a set of labelled formulas $M$ and a labelled formula $G$ : $A$, with the notation $\left[B_{j}^{n}\right] M$ we display all the labels of the form $B_{j}$ at the level $n$ that occur in $M$; with the notation $\left[B_{j}^{n}\right] G: A$ we display the label of the form $B_{j}$ at the level $n$ that occur in $G$.

- Given a set of labelled formulas $M$, we will use the notation $B_{j}^{1} ;[M]$ to indicate that each formula $G: A$ belonging to $M$ has been changed into the formula $B_{j}^{1} ; G^{\prime}: A$, where $G^{\prime}$ is obtained from $G$ by adding 1 to each upper index of $G$.

The sequent calculus GCK is composed by the axioms and rules of Figure 2. First of all, let us make some remarks on the counterfactual logical rules of this calculus. The first remark concerns the rule $>L$. The repetition of the principal formula $A>B$ in the premise of the rule only serves to make the rule invertible. This is analogous to the repetition of the formula $\forall x \alpha(x)$ in the premise of the rule which introduces the symbol $\forall$ on the left side of the sequent in some versions of the sequent calculus for first-order classical logic. Note also that the rule $>L$ carries the proviso that the label $C_{j}^{n}$ should already occur in $M$ or in $N$. This condition is essential to ensure the analyticity of the calculus (and thus also proof-search termination). The last remark concerns the rules $>L$ and $>R$. It is easy to understand these rules if we read them in semantics terms. In this perspective, the rule $>R$ says, if read bottom up, that, if the formula $A>B$ is false at a world $i$, then there exists a world $j$ selected by the selection function $f(i, A)$ where the formula $B$ is false; on the other hand, the rule $>L$ tells us, if read bottom-up, that, if the formula $A>B$ is true 
Figure 2: Axioms and rules of the calculus GCK

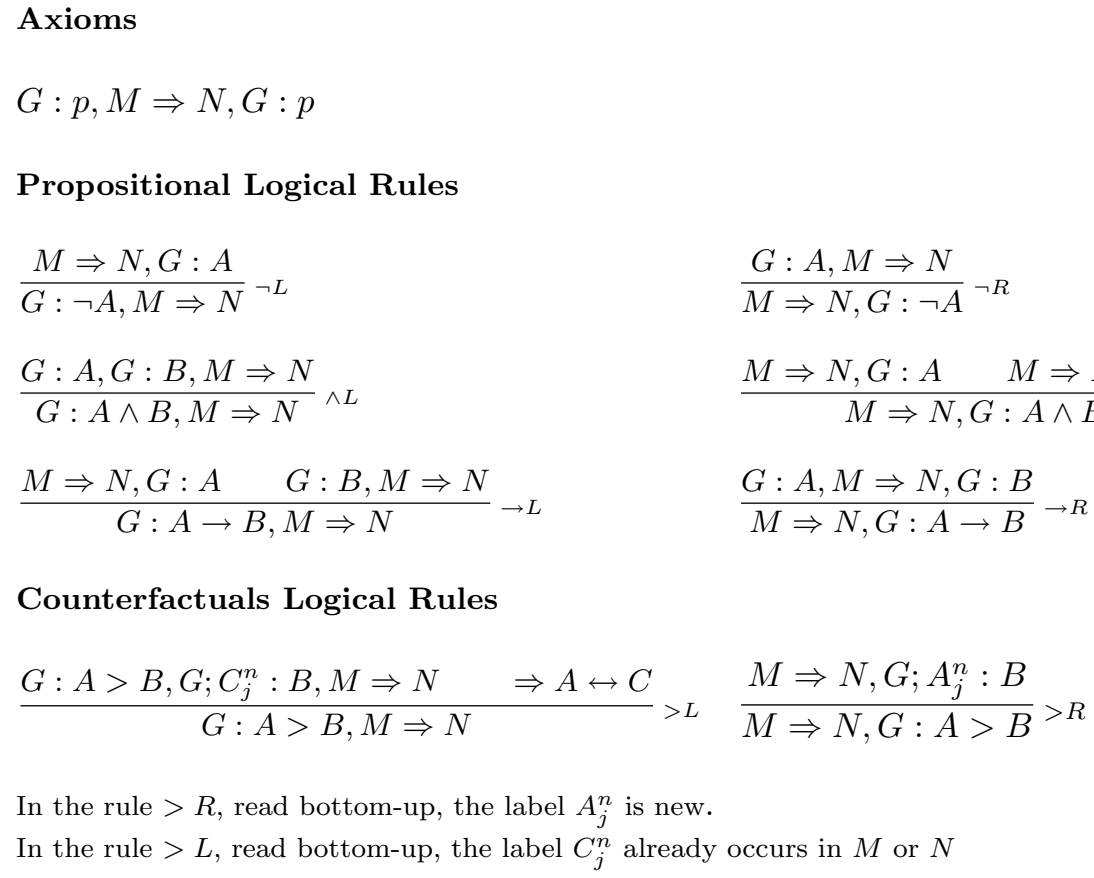

Counterfactuals Logical Rules

$\frac{G: A>B, G ; C_{j}^{n}: B, M \Rightarrow N \quad \Rightarrow A \leftrightarrow C}{G: A>B, M \Rightarrow N}>L \quad \frac{M \Rightarrow N, G ; A_{j}^{n}: B}{M \Rightarrow N, G: A>B}>R$

In the rule $>R$, read bottom-up, the label $A_{j}^{n}$ is new.

In the rule $>L$, read bottom-up, the label $C_{j}^{n}$ already occurs in $M$ or $N$

at a world $i$, then the formula $B$ is true, not only in any world $z$ selected by the selection function $f(i, A)$, but also in any world $j$ selected by the selection function $f(i, C)$, for any formula $C$ such that $A \leftrightarrow C$ is valid in Nute semantics. In other words, while the rule $>R$ reflects the left to right direction of the satisfability relation (see Definition 2.4) for the counterfactual connective, the rule $>L$ not only reflects the right to left direction of the satisfability relation, but it also internalizes the normality condition proper of the selection function of Nute models.

In order to obtain the calculi for the extensions of the system CK, we will consider the rules $i d$ and $m p$ of Figure 3. Note that each of these rules reflects at the proof-theoretic level the semantic condition associated with the axiom ID and the axiom MP, respectively. The sequent calculi for the systems CK* will be the calculi GCK $+i d, \mathrm{GCK}+m p$ and $\mathrm{GCK}+i d+m p$. From now on these calculi, together with the calculus GCK, will be called GCK*.

Definition 3.3. We associate to each derivation $d$ in $\mathrm{GCK}^{*}$ a natural number $h(d)$ (the height). Intuitively, the height corresponds to the length of the longest branch in a derivation $d$, minus one. For the standard definition of the height of a derivation see [22]. 
Figure 3: ID and MP extensions of the calculus GCK

$$
\frac{G ; A_{j}^{n}: A, M \Rightarrow N}{M \Rightarrow N} \text { id } \quad \frac{G: A>B, M \Rightarrow N, G: A \quad G: A>B, G: B, M \Rightarrow N}{G: A>B, M \Rightarrow N}{ }_{m p}
$$

Definition 3.4. $d \vdash_{X}^{m} M \Rightarrow N$ means that $d$ is a derivation of $M \Rightarrow N$ in a calculus $\mathrm{X} \in \mathrm{GCK}^{*}$ with $h(d) \leq m$. We write $\vdash^{\langle m\rangle} M \Rightarrow N$ for: there exists a derivation $d$ such that $d \vdash^{m} M \Rightarrow N$ in $\mathrm{GCK}^{*}$.

Lemma 3.5. Sequents of the form $G: A, M \Rightarrow N, G: A$, with $A$ an arbitrary formula, are derivable in $G C K^{*}$.

Proof. By straightforward induction on G:A.

Lemma 3.6. The rule of substitution:

$$
\begin{aligned}
& M \Rightarrow N \\
& {\left[A_{i}^{n} / A_{j}^{n}\right] M \Rightarrow N\left[A_{i}^{n} / A_{j}^{n}\right] } s u b
\end{aligned}
$$

is height-preserving admissible in $G C K^{*}{ }^{3}$

Proof. By induction on the height of the derivation of the premise. If the premise is an axiom, then so is the conclusion. If the premise is inferred by a rule $\mathcal{R}$, which is either one of the propositional logical rules or one of the rules $>L$, $i d, m p$, then we apply the inductive hypothesis to the premise and then apply $\mathcal{R}$ again. If the premise is inferred by the rule $>R$, we consider the following more peculiar case:

$$
\frac{\langle m-1\rangle M \Rightarrow N, G ; A_{j}^{n}: B}{\langle m\rangle M \Rightarrow N, G: A>B}>R \quad 4_{\rightsquigarrow} \quad \frac{\langle m-1\rangle M \Rightarrow N, G ; A_{k}^{n}: B}{\frac{\langle m-1\rangle\left[A_{i}^{n} / A_{j}^{n}\right] M \Rightarrow N\left[A_{i}^{n} / A_{j}^{n}\right], G ; A_{k}^{n}: B}{\langle m\rangle\left[A_{i}^{n} / A_{j}^{n}\right] M \Rightarrow N\left[A_{i}^{n} / A_{j}^{n}\right], G: A>B}>R}
$$

Lemma 3.7. The rule:

$$
\frac{M \Rightarrow N}{C_{k}^{1} ;[M] \Rightarrow C_{k}^{1} ;[N]}+
$$

\footnotetext{
${ }^{3}$ In accordance with the syntactic notation introduced previously, $\left[A_{i}^{n} / A_{j}^{n}\right] M\left(\left[A_{i}^{n} / A_{j}^{n}\right] N\right)$ stands for: substitute all the labels of the form $A_{j}$ that occur in $M(N)$ at the level $n$ with labels of the form $A_{i}$

${ }^{4}$ The symbol $\rightsquigarrow$ means: the premise of the right side is obtained by induction hypothesis on the premise of the left side.
} 
is height-preserving admissible in $G C K^{*}$.

Proof. By induction on the height of the derivation of the premise. If the premise is an axiom, then so is the conclusion. If the premise is inferred by a propositional logical rule, then the inference is clearly preserved. We give an example using the logical rule $\neg R$ :

$$
\frac{\langle m-1\rangle G: A, M \Rightarrow N}{\langle m\rangle M \Rightarrow N, G: \neg A} \neg R \quad \rightsquigarrow \quad \frac{\langle m-1\rangle C_{k}^{1} ; G^{\prime}: A, C_{k}^{1} ;[M] \Rightarrow C_{k}^{1} ;[N]}{\langle m\rangle C_{k}^{1} ;[M] \Rightarrow C_{k}^{1} ;[N], C_{k}^{1} ; G^{\prime}: \neg A} \neg R
$$

If the premise is inferred by one of the counterfactuals rules, then the inference is preserved. We give an example using the rule $>R$ :

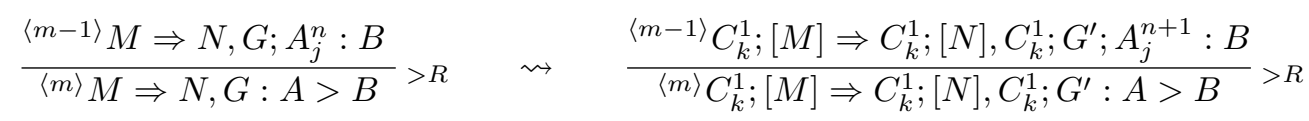

If the premise is inferred by the rules $i d$ or $m p$, then the procedure is analogous to the one of the cases above: we apply the inductive hypothesis on their premise and then we apply $i d$ or $m p$.

Lemma 3.8. The rule of weakening:

$$
\frac{M \Rightarrow N}{M, P \Rightarrow N, Q} W
$$

is height-preserving admissible in $G C K^{*}$.

Proof. By straightforward induction on the height of the derivation of the premise. In case the last applied rule is $>R$, there might be a clash of variables. If so, we first use the rule $s u b$, which is height-preserving admissible, and then we apply the inductive hypothesis.

Lemma 3.9. The propositional logical rules, the counterfactual rules and the rules id and $m p$ are height-preserving invertible.

Proof. The proof proceeds by induction on the height of the derivation of the premise of the rule considered. The cases of the propositional logical rules are dealt with in the classical way. The only differences - the fact that we are dealing with labelled formulas and the cases where the last applied rule is one of the rules $>R,>L, i d$ or $m p$ - are dealt with easily.

The rules $>L, i d$ and $m p$ are trivially height-preserving invertible since both their premises are obtained by weakening from their respective conclusions, and weakening is height-preserving admissible.

We show in detail the invertibility of the rule $>R$. If $M \Rightarrow N, G: A>B$ is an axiom, then so is $M \Rightarrow N, G ; A_{j}^{n}: B$. If $M \Rightarrow N, G: A>B$ has been 
obtained by a propositional logical rule $\mathcal{R}$, we apply the inductive hypothesis on the premise(s), $M^{\prime} \Rightarrow N^{\prime}, G: A>B\left(M^{\prime \prime} \Rightarrow N^{\prime \prime}, G: A>B\right)$ and we obtain derivation(s), of height $m-1$, of $M^{\prime} \Rightarrow N^{\prime}, G ; A_{j}^{n}: B\left(M^{\prime \prime} \Rightarrow N^{\prime \prime}, G ; A_{j}^{n}: B\right)$. By applying the rule $\mathcal{R}$, we obtain a derivation of height $m$ of $M \Rightarrow N, G ; A_{j}^{n}: B$. If $M \Rightarrow N, G: A>B$ is of the form $H: D>C, M^{\prime} \Rightarrow N, G: A>B$, then it may have been obtained by either the counterfactual rule $>L$ or by the rule $m p$. Since the procedure is the same in both cases, we just analyze the case of the rule $>L$; the case of the rule $m p$ can be dealt with analogously. We apply the inductive hypothesis on $H: D>C, H ; E_{z}^{k}: C, M^{\prime} \Rightarrow N, G: A>B$ and we obtain a derivation of height $m-1$ of $H: D>C, H ; E_{z}^{k}: C, M^{\prime} \Rightarrow$ $N, G ; A_{j}^{n}: B$. By applying the rule $>L$, we obtain a derivation of height $m$ of $H: D>C, M^{\prime} \Rightarrow N, G ; A_{j}^{n}: B$. If $M \Rightarrow N, G: A>B$ has been obtained by the rule $i d$ or by the rule $>R$ (in case of the rule $>R$, the labelled formula $G: A>B$ is not the principal formula), then the procedure is analogous to the cases above. Finally, if $M \Rightarrow N, G: A>B$ has been obtained by the counterfactual rule $>R$ and $G: A>B$ is the principal formula, the premise of the last step gives the conclusion.

Lemma 3.10. The rules of contraction:

$$
\frac{G: A, G: A, M \Rightarrow N}{G: A, M \Rightarrow N} C L_{C} \quad \frac{M \Rightarrow N, G: A, G: A}{M \Rightarrow N, G: A} C R
$$

are height-preserving admissible in $G C K^{*}$.

Proof. By induction on the height of the derivation of the premise $M \Rightarrow N, G$ : $A, G: A(G: A, G: A, M \Rightarrow N)$. We only analyze the case of the rule $C R$. The case of the rule $C L$ is symmetric.

If $M \Rightarrow N, G: A, G: A$ is an axiom, so is $M \Rightarrow N, G: A$. If $M \Rightarrow N, G$ : $A, G: A$ is the conclusion of a rule $\mathcal{R}$ which does not have either of the two occurrences of the formula $G: A$ as principal, we apply the inductive hypothesis on the premise(s) $M^{\prime} \Rightarrow N^{\prime}, G: A, G: A\left(M^{\prime \prime} \Rightarrow N^{\prime \prime}, G: A, G: A\right)$, obtaining derivation(s) of height $m-1$ of $M^{\prime} \Rightarrow N^{\prime}, G: A\left(M^{\prime \prime} \Rightarrow N^{\prime \prime}, G: A\right)$. By applying the rule $\mathcal{R}$ we obtain a derivation of height $m$ of $M \Rightarrow N, G: A$.

$M \Rightarrow N, G: A, G: A$ is the conclusion of a propositional logical rule or of the rule $>R$ and one of the two occurrences of the formula $G: A$ is principal. We analyze in detail the following three cases:

$\neg R:$

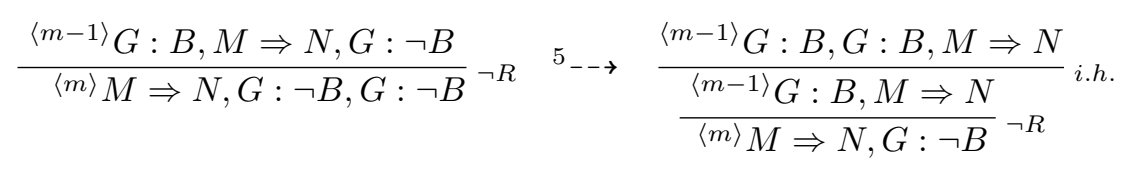

${ }^{5}$ The symbol $\rightarrow$ means: the premise of the right side is obtained by an application of Lemma 3.9 on the premise of the left side. 
$\wedge R$ :

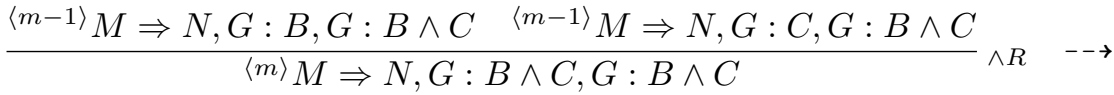

$$
\begin{aligned}
& \frac{\frac{\langle m-1\rangle}{{ }^{\langle m-1\rangle} M \Rightarrow N, G: B, G: B}{ }_{i . h} \quad \frac{\langle m-1\rangle M \Rightarrow N, G: C, G: C}{\langle m-1\rangle M \Rightarrow N, G: C} i . h .}{\langle m\rangle M \Rightarrow N, G: B \wedge C} \wedge R
\end{aligned}
$$

$>R$ :

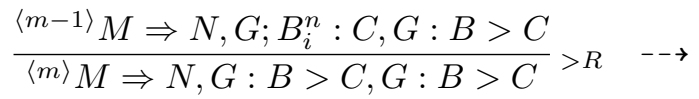

$$
\begin{aligned}
& \frac{{ }^{\langle m-1\rangle} M \Rightarrow N, G ; B_{i}^{n}: C, G ; B_{j}^{n}: C}{{\frac{\langle m-1\rangle}{} M \Rightarrow N, G ; B_{i}^{n}: C, G ; B_{i}^{n}: C}^{\langle m-1\rangle} M \Rightarrow N, G ; B_{i}^{n}: C} \text { sub }
\end{aligned}
$$

\section{The Adequateness Theorem}

In this section we prove that the sequent calculi GCK* are sound and complete with respect to Nute semantics.

Let us denote with $\mathfrak{i d}$ and $\mathfrak{m} \mathfrak{p}$ the semantic properties of Nute frames that correspond to the axioms ID and MP, respectively. Let $\mathrm{X} \subseteq\{\mathfrak{i} \mathfrak{i}, \mathfrak{m p}\} ; \mathfrak{C}+\mathrm{X}$ will denote the class of Nute frames that satisfy the property(ies) denoted by $X$.

Theorem 4.1. For all sequents $M \Rightarrow N$, if $M \Rightarrow N$ is provable in a calculus $X \in G C K^{*}$, then $M \Rightarrow N$ is valid in the corresponding class of frame $\mathfrak{C}+X$, $X \in\{\mathfrak{i d}, \mathfrak{m} \mathfrak{p}\}$.

Proof. By induction on the derivation of the premise. The validity of the axiom and of the propositional rules is proved in the usual way. We omit the proof of the validity of the counterfactual rules, as well as that of the rules $i d$ and $m p$, which is easy but quite tedious.

In order to prove completeness for the calculi GCK*, we will follow a standard technique (e.g. see [3, 19]) and work with different but equivalent calculi that we are going to call $\left(\mathrm{GCK}^{*}\right)^{+}$and which are defined as follows. For each rule $\mathcal{R}$ of $\mathrm{GCK}^{*}$, we define a rule $\mathcal{R}^{+}$which keeps the main formula from the conclusion. For the rules $>L$ and $m p$ we have $\mathcal{R}=\mathcal{R}^{+}$. For the other rules we have 


$$
\begin{aligned}
& \frac{G: \neg A, M \Rightarrow N, G: A}{G: \neg A, M \Rightarrow N}{ }_{(\neg L)^{+}} \quad \frac{G: A, M \Rightarrow N, G: \neg A}{M \Rightarrow N, G: \neg A}(\neg R)^{+} \\
& {\frac{G: A, G: B, G: A \wedge B, M \Rightarrow N}{G: A \wedge B, M \Rightarrow N}(\wedge L)^{+}}_{\underbrace{}_{(\wedge R)^{+}}}
\end{aligned}
$$

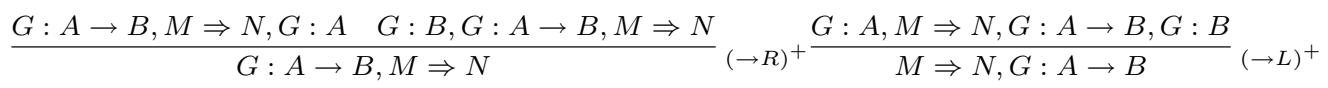$$
\frac{M \Rightarrow N, G: A>B, G ; A_{j}^{n}: B}{M \Rightarrow N, G: A>B}(>R)^{+} \quad \text { where the label } A_{j}^{n}
$$$$
\text { is new, reading the }
$$$$
\text { rule bottom-up. }
$$

Definition 4.2. The set sequent of the sequent $M \Rightarrow N$ is the object $P \Rightarrow Q$, where $P$ and $Q$ are the corresponding sets of the multisets $M$ and $N$, respectively. Clearly the set sequent of a sequent is still a sequent since a set is a multiset.

Let us try to clarify the idea of set sequent by means of an example. Suppose $A, A, B \Rightarrow C, D$ is the sequent $M \Rightarrow N$. Then $A, B \Rightarrow C, D$ is the set sequent $P \Rightarrow Q$.

Any rule $\mathcal{R}^{+}$carries the proviso that for all of its premises the set sequent is different from the set sequent of the conclusion.

Given a calculus $\mathrm{X} \in \mathrm{GCK}^{*}$, the calculus $(\mathrm{X})^{+}$is obtained by replacing each rule $\mathcal{R}$ of $\mathrm{X}$ by the corresponding rule $(R)^{+}$. The calculi $\mathrm{GCK}^{*}$ and $\left(\mathrm{GCK}^{*}\right)^{+}$ happen to be equivalent. For now we prove the following.

Lemma 4.3. For all sequents $M \Rightarrow N$, for all $X \in G C K^{*}$ and all $X^{+} \in$ $\left(G C K^{*}\right)^{+}$,

$$
\text { if } \vdash_{X+} M \Rightarrow N \text { then } \vdash_{X} M \Rightarrow N
$$

Proof. By straightforward induction on the height of derivations in $\left(\mathrm{GCK}^{*}\right)^{+}$, using contraction.

Definition 4.4. A sequent is said to be finished if no rule $\mathcal{R}^{+}$can be applied to one of its labelled formulas.

Definition 4.5. We define a procedure prove $\left(M \Rightarrow N, \mathrm{X}^{+}\right)$, which takes a sequent $M \Rightarrow N$ and a calculus $\mathrm{X}^{+} \in\left(\mathrm{GCK}^{*}\right)^{+}$, and builds a derivation tree for $M \Rightarrow N$ by applying rules from that calculus to non-initial and unfinished derivation leaves (i.e. leaves that do not have an axiomatic form and contain formulas that one can apply rules of the calculi to) in a bottom-up fashion, as follows: 
1. keep applying all the rules of $\mathrm{X}^{+}$which are not the rule $(>R)^{+}$as long as possible;

2. wherever possible, apply the rule $(>R)^{+}$once. $^{6}$

Repeat this operation until each non-initial derivation leaf of the sequent $M \Rightarrow$ $N$ is finished. If $\operatorname{prove}\left(M \Rightarrow N, \mathrm{X}^{+}\right)$terminates and all derivation leaves are initial then it succeeds; otherwise, i.e. if it terminates and there is a non initial derivation leaf, it fails.

Lemma 4.6. For all calculi $\left(G C K^{*}\right)^{+}$and for all sequents $M \Rightarrow N$, the procedure $\operatorname{prove}\left(M \Rightarrow N, X^{+}\right)$always terminates.

Proof. Each of the rules $R^{+}$causes the set of the labelled formulas of the sequent $M \Rightarrow N$ to strictly grows. By the subformula property, only finitely many labelled formulas can occur in a sequent, so the procedure terminates. Note that both rules $i d$ and $>L$ might be applied several times: the rule $i d$ will be applied once for each new label introduced, the rule $>L$ will be applied on each labelled formula of the form $G ; C_{z}^{n-1}: A>B$ occurring on the left side of the sequent as many times as there are labels $E_{j}^{n}$, such that $E \leftrightarrow A$. However, since the number of labelled formulas and hence of their labels is finite, the rules $i d$ and $>L$ will only be applied a finite number of times.

In order to prove the completeness theorem for our $\left(\mathrm{GCK}^{*}\right)^{+}$calculi, we need the following two definitions.

Definition 4.7. Let $f$ be a function in a Nute model, which, therefore, associates to each world and each formula a set of possible worlds. We indicate with $f^{i d}$ the function in a Nute model that satisfies the semantic condition id; we indicate with $f^{m p}$ the function in a Nute model that satisfies the semantic condition $\mathfrak{m p}$. $f^{i d+m p}$ will denote the function in a Nute model that satisfies both semantic conditions $\mathfrak{i d}$ and $\mathfrak{m} \mathfrak{p}$. Let $\mathrm{X} \subseteq\{\mathfrak{i} \mathfrak{d}, \mathfrak{m} \mathfrak{p}\}$, then $f^{X}$ is the function that satisfies the property(ies) of X.

Definition 4.8. Let us consider the $n$ labelled formulas that occur in a sequent $M \Rightarrow N$. We divide these labelled formulas in sets $S_{1}, \ldots, S_{m}$ in the following way. Each set $S_{i}, 1 \leq i \leq m$, will contain all and only those labelled formulas of $M$ and $N$ that share the same sequence of labels $G$. We call these sets label sets.

Let $S_{i}$ be a label set. With $S_{i}^{*}$ we will indicate the set composed by those formulas obtained by erasing from each labelled formula contained in $S_{i}$ its label-sequence $G$. Sets as $S_{i}^{*}$ will be called label-free sets.

Theorem 4.9. For all $X \subseteq\{\mathfrak{i d}, \mathfrak{m p}\}$ and all sequents $M \Rightarrow N$, we have that

\footnotetext{
${ }^{6}$ Since the rule $(>R)^{+}$creates new labels, in order not to have loops, it should only be applied once.
} 
(i) if $M \Rightarrow N$ is valid with respect to a $\mathfrak{C}+X$-frame, then the sequent calculus $X \in G C K^{*}$ that corresponds to the $\mathfrak{C}+X$-frame is such that $\vdash$ $M \Rightarrow N$ in $X$.

(ii) If $\operatorname{prove}\left(M \Rightarrow N,(X)^{+}\right)$fails, then $M \Rightarrow N$ is not valid in the frame that corresponds to the calculus $(X)^{+}$.

Proof. The contrapositive of (i) follows from (ii). If $\nvdash M \Rightarrow N$ in a calculus $\mathrm{X} \in \mathrm{GCK}^{*}$, then by Lemma 4.3 , also $\nvdash M \Rightarrow N$ in the corresponding calculus $(\mathrm{X})^{+} \in\left(\mathrm{GCK}^{*}\right)^{+}$; thus, in particular, prove $\left(M \Rightarrow N,(\mathrm{X})^{+}\right)$cannot yield a derivation and by Lemma 4.6 has to fail. For (ii), we define a model $\mathfrak{M}$ on a $\mathfrak{C}$ $+X$-frame, for $X \subseteq\{\mathfrak{i} \mathfrak{d}, \mathfrak{m} \mathfrak{p}\}$, for which we prove that it is a countermodel for $M \Rightarrow N$.

Let $P \Rightarrow Q$ be a non-initial sequent obtained by the procedure $\operatorname{prove}(M \Rightarrow$ $\left.N,(\mathrm{X})^{+}\right)$. Let $S_{1}, \ldots, S_{m}$ be the label sets that can be obtained from $P \Rightarrow Q$ and $S_{1}^{*}, \ldots, S_{m}^{*}$ the corresponding label-free sets. $\mathrm{W}=\left\{S_{1}^{*}, \ldots, S_{m}^{*}\right\}$. The function $f^{X}$ is explicitly defined by the labels contained in $S_{1}, \ldots, S_{m}$. We define $v$ in the following way

$$
v\left(S_{i}^{*}, p\right)=\left\{\begin{array}{cl}
1: & p \text { occurs in a label formula } G: p \text { such that } G: p \in S_{i} \\
& \text { and occurs on the left side of the sequent } P \Rightarrow Q
\end{array} \quad \begin{array}{l}
p \text { occurs in a label formula } G: p \text { such that } G: p \in S_{i} \\
\text { and occurs on the right side of the sequent } P \Rightarrow Q
\end{array}\right.
$$

Since the sequent $P \Rightarrow Q$ is non-initial, this choice can be coherently made. We thus have $\mathfrak{M}=\left\langle W, f^{X}, v\right\rangle$.

Claim 1. For all $S_{i}^{*}, S_{j}^{*} \in W$ such that $S_{j}^{*} \in f\left(S_{i}^{*}, A\right)$, for some $A$, and for all labelled formulas of the form $G: B>C$, such that they occur on the left side of the sequent $P \Rightarrow Q$ and $\models B \leftrightarrow A$ is valid in Nute semantics, we have that, if $B>C \in S_{i}^{*}$, then $C \in S_{j}^{*}$.

By the $>L$ rule we have that $C$ is in all $S_{j}^{*}$ such that $S_{j}^{*} \in f\left(S_{i}^{*}, A\right)$, for all $A$ such that $B \leftrightarrow A$ is a validity.

Claim 2. For all $S_{i}^{*} \in W$ and for all labelled formulas of the form $G: B>C$, such that they occur on the left side of the sequent $P \Rightarrow Q$, we have that, whenever $\mathfrak{m p} \in X$ in $f^{X}$, if $B>C \in S_{i}^{*}$, then $B$ or $C \in S_{i}^{*}$ as well.

The application of the rule $m p$ easily allows to verify Claim 2 .

Claim 3. For all $S_{i}^{*} \in W$, we have:

- for all $A \in S_{i}^{*}$ such that $G: A$ occur on the left side of the sequent $P \Rightarrow Q$, $S_{i}^{*} \models_{\mathfrak{M}} G: A$

- for all $A \in S_{i}^{*}$ such that $G: A$ occur on the right side of the sequent $P \Rightarrow Q, S_{i}^{*} \not \nvdash_{\mathfrak{M}} G: A$ 
By induction on the complexity of the formula $G: A$. Concerning propositional atoms, it follows from the definition of the valuation function, and the fact that $P \Rightarrow Q$ is not an initial sequent. Concerning the propositional connectives, it is clear from the shape of the $(\neg)^{+}$, the $(\wedge)^{+}$and $(\rightarrow)^{+}$rules. If $G: A=G: B>C$ and it occurs on the right side of the sequent, then by the $(>R)^{+}$rule, we have at least one $S_{z}^{*} \in f^{X}\left(B, S_{k}^{*}\right)$ with $C \in S_{z}^{*}$. By the inductive hypothesis, we have $S_{z}^{*} \nvdash_{\mathfrak{M}} C$ and thus $S_{k}^{*} \nvdash_{\mathfrak{M}} B>C$; hence $S_{i}^{*} \nvdash_{\mathfrak{M}} G: B>C$. If $G: A=$ $G: B>C$ and it occurs on the left side of the sequent, then:

(i) by Claim 1, we have $C \in S_{z}^{*}$ for all $S_{z}^{*} \in f^{X}\left(D, S_{k}^{*}\right)$, for any $D$ such that $B \leftrightarrow D$ is valid in Nute semantics. Since $B \leftrightarrow B$ is a validity, then $C \in$ $S_{z}^{*}$ for all $S_{z}^{*} \in f^{X}\left(B, S_{k}^{*}\right)$. Thus, by the inductive hypothesis, we have $S_{z}^{*}=\mathfrak{M} C$.

(ii) by Claim 2, we have $B$ or $C \in S_{k}^{*}$. Thus, by the inductive hypothesis, we have $S_{k}^{*} \not \nvdash_{\mathfrak{M}} B$ or $S_{k}^{*} \models_{\mathfrak{M}} C$, which is to say: if $S_{k}^{*} \models_{\mathfrak{M}} B$, then $S_{k}^{*} \models_{\mathfrak{M}} C$. C.

By (i) and (ii) we can infer $S_{k}^{*} \models_{\mathfrak{M}} B>C$. Hence we have $S_{i}^{*} \models_{\mathfrak{M}} G: B>$

Claim 4. The selection function $f^{X}$ of the model $\mathfrak{M}=\left\langle W, f^{X}, v\right\rangle$ satisfies the normality condition.

Suppose that the selection function $f^{X}$ of the model $\mathfrak{M}=\left\langle W, f^{X}, v\right\rangle$ does not satisfy the normality condition. This means that there exist a formula $A$ and a formula $C$ such that $A \leftrightarrow C$ is valid in Nute semantics but $f^{X}(i, A) \neq f^{X}(i, C)$. The fact that $f^{X}(i, A) \neq f^{X}(i, C)$ in the model $\mathfrak{M}$ implies that there exist two worlds $S_{i}^{*}, S_{j}^{*} \in W$ and a formula of the form $C>B$ such that: (i) $S_{j}^{*} \in$ $f\left(S_{i}^{*}, A\right)$, (ii) $C>B$ belongs to $S_{i}^{*}$ and $G: C>B$ occurs on the left side of the sequent $P \Rightarrow Q$, (iii) the formula $B$ does not belong to $S_{j}^{*}$.

Since the formula $G: C>B$ occurs on the left side of the sequent $P \Rightarrow Q$, $C>B$ belongs to $S_{i}^{*}$ and $A \leftrightarrow C$ is a validity, by Claim 1, we can infer that the formula $C$ belongs to any world $S_{z}^{*}$ such that $S_{z}^{*} \in f\left(S_{i}^{*}, A\right)$; thus $C$ also belongs to $S_{j}^{*}$. This contradicts (iii).

Claim 5. If $\mathfrak{i} \in \mathrm{X}$, then the selection function $f^{X}$ of the model $\mathfrak{M}=\left\langle W, f^{X}, v\right\rangle$ satisfies the $\mathfrak{i d}$ condition.

Thanks to the applications of the rule $i d$ we know that for all $S_{i}^{*}$ and for all $S_{j}^{*}$ such that $S_{j}^{*} \in f\left(S_{i}^{*}, A\right)$, for some $A, A \in S_{j}^{*}$, moreover $G ; A_{j}^{n}: A$ occurs on the left side of the sequent $P \Rightarrow Q$. By Claim 3 we have $S_{j}^{*}=_{\mathfrak{M}} A$.

The model $\mathfrak{M}$ satisfies all the required properties. Since all rules seen top-down preserve countermodels, Claim 3 implies that $\nvdash_{\mathfrak{M}} M \Rightarrow N$.

\section{$5 \quad$ Natural Deduction Calculi}

We use this section to introduce natural deduction calculi for the $\mathrm{CK}^{*}$ systems. In order to build these calculi we will use the same technique used for the 
Figure 4: Rules of the calculus NCK

\begin{tabular}{|c|c|}
\hline Introduction Rules & Elimination Rules \\
\hline \multicolumn{2}{|l|}{ Propositional Rules } \\
\hline$[G: A]$ & {$[G: A]$} \\
\hline $\begin{array}{l}\vdots d \\
\perp\end{array}$ & $\vdots d$ \\
\hline$\frac{\perp}{G: \neg A} \neg I$ & $\frac{\perp}{G: A} \neg E$ \\
\hline$d_{1} \quad d_{2}$ & $d$ \\
\hline$\frac{\dot{G}: A \quad \dot{G}: B}{G: A \wedge B} \wedge I$ & $\frac{G: \dot{A} \wedge B}{G: A} \wedge E \quad \frac{G: \dot{A} \wedge B}{G: A} \wedge E$ \\
\hline \multicolumn{2}{|l|}{$[G: A]$} \\
\hline$\frac{\vdots d}{G: B}=$ & $\frac{G: \dot{A} \rightarrow B \quad \dot{G}: A}{G: B} \rightarrow E$ \\
\hline \multicolumn{2}{|l|}{ Counterfactual Rules } \\
\hline$d$ &..$d_{2}$ \\
\hline$\frac{G ; \dot{A}_{j}^{n}: B}{G: A>B}>I$ & 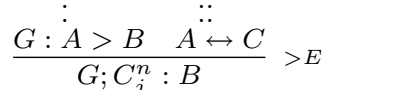 \\
\hline \multicolumn{2}{|c|}{$\begin{array}{l}\text { In the rule }>I \text { the label } A_{j}^{n} \text { does not occur in any undischarged assumption of } d \text {. } \\
\text { In the rule }>E \text { the label } C_{j}^{n} \text { either already occurs in the derivation } d_{1} \text { or it will } \\
\text { be the principal label of an application of the rule }>I \text {. }\end{array}$} \\
\hline
\end{tabular}

sequent calculi, namely we will employ labelled formulas. The natural deduction calculus NCK for the system CK is composed of the rules of Figure 4. As usual, the rules are divided into introduction and elimination rules. As for the rules that introduce and eliminate negation, conjunction and implication, these are the classical ones, the only difference being that we use labelled formulas instead of standard formulas. As for the rules that introduce and eliminate the counterfactual symbol, these are analogous to the $>L$ and $>R$ rules of the sequent calculus GCK, respectively. Let us draw the reader's attention on the special notation used in the derivation of the formula $A \leftrightarrow C$ in the rule $>E$. This notation serves to indicate the fact that in the derivation of the formula $A \leftrightarrow C$ all assumptions must be discharged.

In order to take into account the axioms ID and MP, we will consider the rules of Figure 5. Thus, the natural deduction calculi for the systems $\mathrm{CK}^{*}$ will be the calculi NCK, NCK+nid, NCK+nmp and NCK+nid+nmp. From now 
Figure 5: ID and MP extensions of the calculus NCK

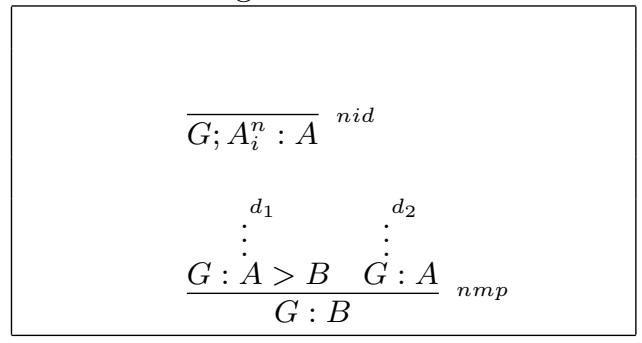

on these calculi will be referred to as $\mathrm{NCK}^{*}$.

Before turning to the introduction of some important definitions and lemmas, let us illustrate some derivations in the calculi $\mathrm{NCK}^{*}$.

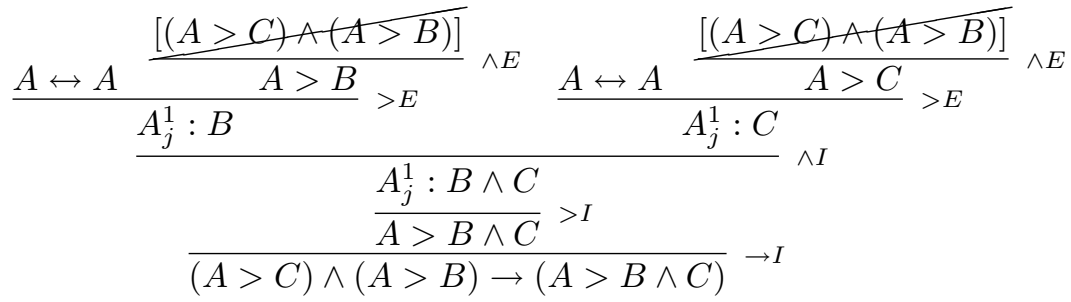

$$
\begin{aligned}
& \frac{\left[A_{j}^{1}: \neg \text { B } \frac{A \leftrightarrow A \quad A>B}{A_{j}^{1}: B}\right.}{\frac{\perp}{A_{j}^{1}: \neg \neg B} \neg_{I}^{A}>I}>E \\
& \frac{A \leftrightarrow \neg \neg A \quad A>C}{\neg \neg A_{j}^{1}: C}>E \\
& \overline{\neg \neg A>C}>I \\
& \frac{\overline{\neg \neg A>C \vee \neg \neg A>D} \vee I}{(A>C) \rightarrow(\neg \neg A>C \vee \neg \neg A>D)} \rightarrow I
\end{aligned}
$$

Definition 5.1. The notions of deduction and height of a deduction in $\mathrm{NCK}^{*}$ are the standard ones, see [18]. We write $M \vdash_{X}^{m} G: A$ for: there exists a deduction $d$ from the multiset $M$ to $G: A$ in a calculus $\mathrm{X} \in \mathrm{NCK}^{*}$ with the height of $d, h(d), \leqslant m$. 
Lemma 5.2. For all $X \in N C K^{*}$, for all multiset $M$ and formula $G: B$, if $M \vdash_{X}^{m} G: B$, then $\left[A_{i}^{n} / A_{j}^{n}\right] M \vdash_{X}^{m}\left[A_{i}^{n} / A_{j}^{n}\right] G: B$.

Proof. By induction on the height of the deduction of $G: B$ from $M$. We distinguish cases according to the last rule $\mathcal{R}$ applied in the deduction $M \vdash_{X}^{m}$ $G: B$. If there is no such a rule $\mathcal{R}$, since $G: B$ is an assumption, then so is $\left[A_{i}^{n} / A_{j}^{n}\right] G: B$. If the last rule $\mathcal{R}$ applied in the deduction $M \vdash_{X}^{m} G: B$ is one of the propositional rules or one of the rules $>E$, nid, nmp, then we apply the inductive hypothesis on the premise of $\mathcal{R}$ and then apply $\mathcal{R}$ again.

If the last rule $\mathcal{R}$ applied in the deduction $M \vdash_{X}^{m} G: B$ is $>I$, then we consider the following specific case:

$$
\begin{aligned}
& \text { M }
\end{aligned}
$$

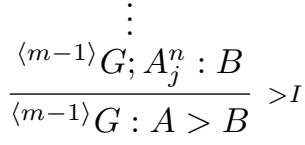

We apply twice the inductive hypothesis on the deduction $M \vdash_{X}^{m-1} G ; A_{j}^{n}: B$ obtaining $\left[A_{i}^{n} / A_{j}^{n}\right] M \vdash_{X}^{m-1} G ; A_{k}^{n}: B$. The application of the rule $>I$ gives the desired conclusion.

Lemma 5.3. For all $X \in N C K^{*}$, for all multiset $M$ and formula $G: B$, if $M \vdash_{X}^{m} G: B$, then $C_{j}^{1} ;[M] \vdash_{X}^{m} C_{j}^{1} ; G^{\prime}: B$.

Proof. By a straightforward induction on the height of the deduction of $G: B$ from $M$.

Lemma 5.4. For all $X \in N C K^{*}$, for all multiset $M$ and formula $G: B$, if $M \vdash_{N C K *}^{m} G: B$, then $M, N \vdash_{N C K *}^{m} G: B$.

Proof. It follows from the definition of deduction employed in the calculi. In case of clash of variables with the rule $>I$, we use Lemma 5.2.

Lemma 5.5. For all $X \in N C K^{*}$, for all multiset $M$ and formula $G: B$, if $H: C, H: C, M \vdash_{X}^{m} G: B$, then $H: C, M \vdash_{X}^{m} G: B$.

Proof. Contraction is achieved by identifying two distinct labels for assumption classes containing $H: C$, respectively.

\section{Normalization Theorem}

We use this section to prove that in natural deduction calculi NCK* deductions normalize. Let us first introduce some important definitions and lemmas.

Definition 6.1. A maximal segment of lenght 1 , or a cut, in a deduction $d$ of a calculus $\mathrm{X} \in \mathrm{NCK}^{*}$ is a labelled formula $G: A$ which is, at the same time, the conclusion of an introduction rule and the major premise of an elimination rule. 
The cutrank of a cut, or of a maximal segment, $G: A$ is $|A|$. The cutrank of a deduction $d$ is the maximum of the cutranks of cuts in $d$. If in $d$ there is no cut, then the cutrank of $d$ is zero. A critical cut of $d$ is a cut of maximal cutrank among all cuts in $d$. A deduction without critical cut is said to be normal.

Lemma 6.2. For all $X \in N C K^{*}$, if $M \vdash_{X} G: A$, then there is a deduction $d^{\prime}$ in $X \in N C K^{*}$ of $G: A$ from $M$ where the consequence of every application of the rule $\neg E$ is either atomic or it can be eliminated.

Proof. Let $d$ be a deduction in $\mathrm{NCK}^{*}$ of $G: A$ from $M$ in which the highest complexity of a consequence of an application of the $\neg E$ rule is $m$, where $m>0$. Let $H: B$ be a consequence of an application $\alpha$ of the $\neg E$ rule in $d$ such that its degree is $m$ but no consequence of an application of the $\neg E$-rule that stands above $H: B$ is of degree $m$. Then $d$ has the form:

$$
\begin{gathered}
\frac{[H: B]}{\frac{d_{2}}{\perp}} \\
\frac{(H: B)}{d_{1}}
\end{gathered}
$$

where $[H: \neg B]$ is the set of assumptions discharged by $\alpha . H: B$ has one of the shapes $H: \neg C, H: D \wedge C, H: A \rightarrow C, H: D>C$. We remove this application of the $\neg E$ rule by transforming $d$ in the respective cases to:

[Negation case]

$$
\frac{\frac{[H: A] \quad[H: \neg A]}{\frac{\perp}{[H: \neg \neg A]}} \neg I}{\frac{\frac{d_{2}}{\perp}}{H: \neg A} \neg I}
$$

[Conjunction case]

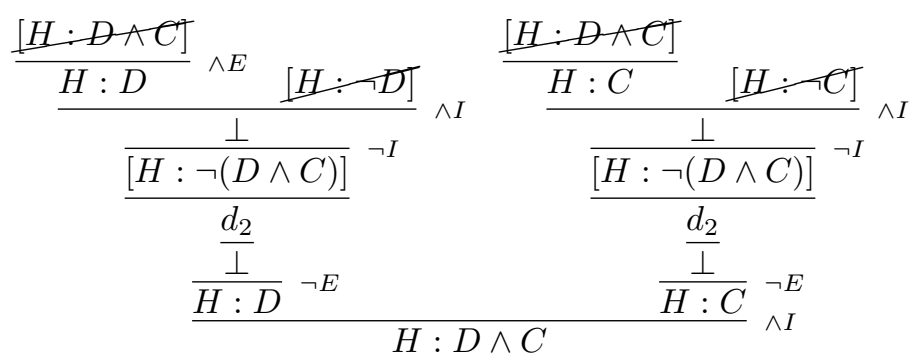

[Implication case] 


$$
\frac{[H: D \rightarrow C] \quad \frac{[H: D]}{[H: C]} \rightarrow E \quad[H: \neg C]}{\frac{\frac{\perp}{[H: \neg(D \rightarrow C)]}}{\frac{[H}{\frac{d_{2}}{\frac{\perp}{H: C} \neg E}}}} \wedge I
$$

[Counterfactual case]

$$
\begin{aligned}
& \frac{\left[H ; D_{i}^{n} \cdot \neg C\right] \frac{[H: D>C] \quad D \stackrel{:}{\leftrightarrow}}{H ; D_{i}^{n}: C}}{\frac{\perp}{\frac{\perp H: \neg(D>C)]}{\frac{d_{2}}{L}}}} \neg I \\
& \frac{\frac{\frac{1}{\perp}}{H ; D_{i}^{n}: C}}{H: D>C}>I
\end{aligned}
$$

We firstly show how to remove propositional cuts which are common to all calculi NCK* ${ }^{*}$. Then we show how to remove counterfactual cuts and finally cuts generated by the rule $n m p$. These last two types of cut are both not so simple to be dealt with and require a more detailed treatment.

$$
\begin{aligned}
& {[\neg I-\neg E]} \\
& {[G: p]} \\
& d_{1} \\
& \frac{\perp}{G: \neg p} \neg I \\
& d_{2} \\
& \frac{\perp}{G: p} \neg E \\
& d_{3} \\
& \text { cont } \\
& \begin{array}{c}
{[G: \neg p]} \\
d_{2} \\
\frac{\perp}{G: p} \neg E \\
d_{3}
\end{array} \\
& {[\wedge I-\wedge E]}
\end{aligned}
$$




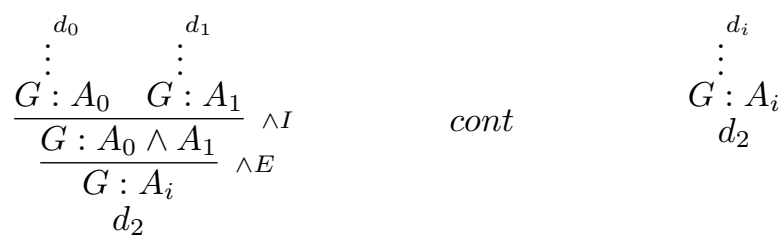

where $i=\{0,1\}$.

$$
\begin{aligned}
& {[\rightarrow I-\rightarrow E]} \\
& \text { [G:A] } \\
& d_{1}
\end{aligned}
$$

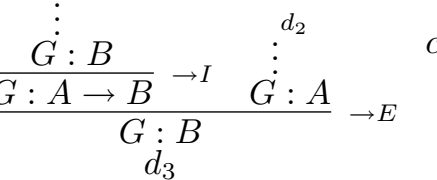

$$
\begin{aligned}
& \text { cont } \\
& \dot{G} \underset{d_{1}}{ } A \\
& \underset{d_{3}}{G} B
\end{aligned}
$$

$[>I->E]$

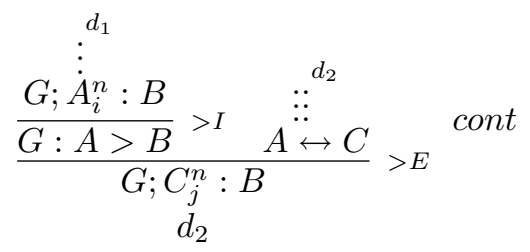

$[>I-n m p]$

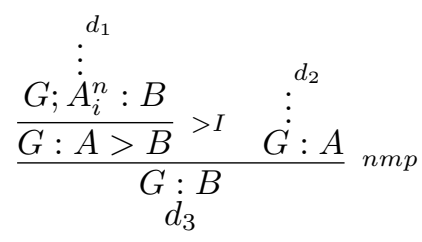

In order to solve this case, we need a lemma stating that, if we have a deduction $d_{1}$ of $G ; A_{i}^{n}: B$ from $M$ and a deduction $d_{2}$ of $G: A$ from $P$, then we have a deduction $d_{3}$ of $G: B$ from $M$ and $P$. In order to prove this lemma, we need the normalization theorem. For not creating any loop, we prove the normalization theorem and the above mentioned lemma by parallel induction. 
In what follows we will prove, by parallel induction, the normalization theorem for the calculi $\mathrm{NCK}^{*}$ together with two lemmas that, as explained above, are required for the very same proof of the normalization theorem. Such a technique has been introduced by [1] for deep-inferences (or tree-hypersequent) calculi for the systems CK* . Here we adapt such a technique to the case of natural deduction calculi with labelled formulas.

We start considering calculi NCK and NCK+nid; successively we will deal with those calculi that contain the $n m p$ rule.

Theorem 6.3. The following propositions hold:

(i) Every derivation d in NCK and NCK+nid is normalizable.

(ii) If $M \vdash_{X} G ; A_{i}^{n}: B$, where the label $A_{i}^{n}$ does not occur in $M$, and there is a norma derivation without undischarged assumptions of $A \leftrightarrow C$ in $X$, then $M \vdash_{X} G ; C_{j}^{n}: B$, where $X$ is either $N C K$ or $N C K+$ nid.

Proof. Let us call $C u t(c, h)$ the assertion that the property (i) holds for the cutrank $c$ and the sum $h$ of the heights of all critical cuts in $d$. Let us call $S u b(c)$ the assertion that (ii) holds for any $M$ and any labelled formula $H: D$ of complexity $c$. We show the following facts:

(i) $\forall c \forall h\left(\forall c^{\prime}<c \operatorname{Cut}\left(c^{\prime}, h\right) \wedge \forall h^{\prime}<h \operatorname{Cut}\left(c, h^{\prime}\right) \rightarrow \operatorname{Cut}(c, h)\right)$

(ii) $\forall c(\forall h C u t(c, h) \rightarrow S u b(c))$.

Let us first prove (i). We will do it by primary induction on the cutrank $c$ and secondary induction on $h$, the sum of the heights of the cuts. By a suitable choice of the critical cuts to which we apply the contraction, we can achieve that either $c$ decreases, or that $c$ remains constant but $h$ decreases. Let us call $\sigma$ a top critical cut in $d$ if no critical cut occurs in a branch of $d$ above $\sigma$. Now apply a contraction to the rightmost top critical cut of $d$; then the resulting $d^{\prime}$ has a lower cutrank, or has a same cutrank but a lower value for $h$.

Let us see this in the case of counterfactual. Suppose we apply a contraction to the rightmost top critical cut consisting of a formula occurrence $G: A>B$

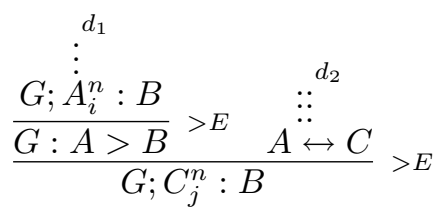

Since $|\mathrm{B}|<|A>B|$, the label $A^{n}$ does not occur in the undischarged assumptions of $d_{1}$, because of the restriction of the rule $>I$, and the derivation $d_{2}$ is in normal form because if it was not, it would contain the rightmost cut against the initial assumption, by inductive hypothesis we can apply (ii) to the derivation $d_{1}$ and the derivation $d_{2}$ obtaining a derivation $d_{3}$ of $G ; C_{j}^{n}: B$ (from the same undischarged assumptions of $d_{1}$ ). This is exactly what we were looking for. 
Before passing to the proof of (ii), let us remind two standard properties of normal deductions which will be useful in what follows.

Theorem 6.4. Let $d$ be a normal deduction in NCK or NCK+nid and let $\beta$ $=G_{1}: A_{1}, G_{2}: A_{2}, \ldots, G_{k}: A_{k}$ be a branch in $d$. Then there is a labelled formula occurrence $G_{i}: A_{i}$, called the minimum formula in $\beta$, which separates two (possibly empty) parts of $\beta$, called the E-part and the I-part of $\beta$, with the properties:

- Each $G_{j}: A_{j}$ in the E-part (i.e. $j<i$ ) is a major premise of an E-rule.

- $G_{i}: A_{i}$, provided that $i \neq k$, is premiss of an I-rule or of the rule $\neg E$.

- Each $G_{j}: A_{j}$ in the I-part, except the last one (i.e. $i<j<k$ ), is a premiss of an I-rule.

Proof. The proof can be developed in the same way as the proof of Theorem 3 in $[18$, p. 41-42].

Corollary 6.5. Let $d$ be a derivation of $G: A$ in $N C K$ or NCK+nid without undischarged assumptions, which is normal and such that $G: A$ is not atomic. Then the derivation $d$ ends with an I-rule.

Proof. The proof can be developed in the same way as the proof of Exercise $6.26 \mathrm{D}$ in $[22$, p. 148].

Let us now pass to (ii). We proceed by induction on the height $h$ of the derivation of $G ; A_{i}^{n}: B$ from $M$. We distinguish cases according to the last rule that has introduced $G ; A_{i}^{n}: B$. If $G ; A_{i}^{n}: B$ is an assumption, $G ; C_{i}^{n}: B$ is an assumption too. If $G ; A_{i}^{n}: B$ has been derived by a rule $\mathcal{R}$ that is either a propositional rule or the rule $>I$, then we apply the inductive hypothesis and then the rule $\mathcal{R}$ again. If $G ; A_{i}^{n}: B$ has been derived by the rule $>E$, then we consider the following problematic case:

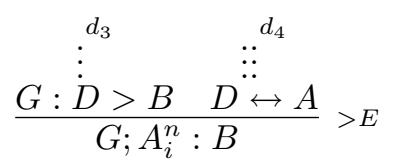

To solve this case, we proceed in the following way. Let us consider the derivation of $A \leftrightarrow C$, let us call this derivation $d_{2}$, and the derivation $d_{4}$ of $D \leftrightarrow$ $A$; both these derivations are normal and without undischarged assumptions. Since $d_{2}$ and $d_{4}$ are normal, do not contain any undischarged assumption and end up with non-atomic formula, we can apply on each of them Corollary 6.5 and thus know that both $d_{2}$ and $d_{4}$ terminate with an introduction rule. Given the form of their last formulas, namely $A \leftrightarrow C$ and $D \leftrightarrow A$, respectively, then in both cases we know that their last rule is the rule $\wedge I$. In particular, we know that the derivation $d_{2}$ ends with $\wedge I$ applied on $A \rightarrow C$ and $C \rightarrow A$, while the derivation $d_{4}$ ends with $\wedge I$ applied on $A \rightarrow D$ and $D \rightarrow A$. 


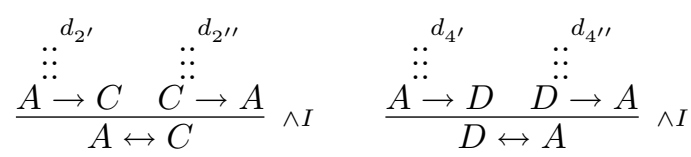

Let us now consider the derivations $d_{2^{\prime}}, d_{2^{\prime \prime}}, d_{4^{\prime}}, d_{4^{\prime \prime}}$. Each of them is in normal form, does not contain open assumptions and ends up with non-atomic formula. Thus, once more, on each of them we can apply Corollary 6.5 and thus know that each of them terminates with an introduction rule. Given the form of their last formulas, then all of $d_{2^{\prime}}, d_{2^{\prime \prime}}, d_{4^{\prime}}, d_{4^{\prime \prime}}$ have as their last rule the rule $\rightarrow I$. In particular each of them is nothing but the result of the application of the rule $\rightarrow I$ to one of the following derivations, respectively:

- a derivation $d^{\prime}$ of $C$ from $A$

- a derivation $d^{\prime \prime}$ of $A$ from $C$

- a derivation $d^{\prime \prime \prime}$ of $D$ from $A$

- a derivation $d^{i v}$ of $A$ from $D$

Let us now consider the derivations $d^{\prime}-d^{i v}$. By combining together $d^{\prime \prime}$ and $d^{\prime \prime \prime}$ and $d^{\prime}$ and $d^{i v}$, we obtain a derivation $d^{*}$ of $D$ from $C$ and a derivation $d^{* *}$ of $C$ from $D$, respectively. By applying on $d^{*}$ and $d^{* *}$ the rules $\rightarrow I$ and $\wedge I$, we obtain:

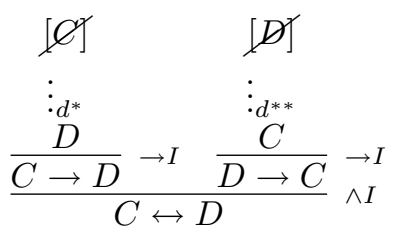

The new derivations $d^{*}$ and $d^{* *}$ might contain contractions the cutrank of which is $|A|$. Since $|A|<|A>B|$, on these contractions we apply (i) and obtain a cut-free derivation of $C \leftrightarrow D$. By applying the rule $>E$ on $G: D>B$ and $C \leftrightarrow D$, we get a normal derivation of $G ; C_{j}^{n}: B$, as desired.

Let us finally suppose that $G ; A_{j}^{n}: B$ is of the form $G ; A_{j}^{n}: A$ and has been obtained by the rule $i d$. We proceed as follows. Let us consider the derivation $d_{2}$ of $A \leftrightarrow C$. Since $d_{2}$ is normal, does not contain any undischarged assumption and ends up with a non-atomic formula, following Corollary 6.5 we know that $d_{2}$ terminates with an introduction rule. Given the form of the last formula of $d_{2}$, namely $A \leftrightarrow C$, the last rule in question will be the rule $\wedge I$. Thus we have:

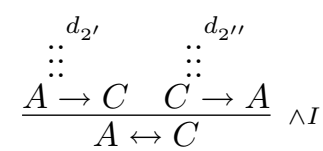

Let us now consider the derivations $d_{2^{\prime \prime}}$ of $C \rightarrow A$. $d_{2^{\prime \prime}}$ is in normal form, does not contain open assumptions and ends up with non-atomic formula. Thus, 
once more, we can apply Corollary 6.5 and thus know that it terminates with an introduction rule. Given the form of its last formula, namely $C \rightarrow A$, then $d_{2^{\prime \prime}}$ has as its last rule the rule $\rightarrow I$. In particular it is nothing but the result of the application of the rule $\rightarrow I$ to a derivation $d^{\prime \prime}$ of $A$ from $C$. Let us consider this derivation $d^{\prime \prime}$; by applying several times Lemma 5.3 on it, we obtain a derivation $d^{*}$ of $G ; C_{i}^{n}: A$ from $G ; C_{i}^{n}: C$. For the rule nid and Lemma 5.4 , we obtain a derivation of $G ; C_{i}^{n}: A$ from $M$ as desired.

Let us now pass to analyze the calculi NCK $+n m p$ and $\mathrm{NCK}+n i d+n m p$. The proof of the normalization theorem is in this case slightly different from the one of Theorem 6.3 above, that is why we prove it in a separate theorem. As previously stated, to make normalization work we need to show the following statement:

If $M \vdash_{X} G ; A_{i}^{n}: B$, where the label $A_{i}^{n}$ does not occur in $M$, and $P \vdash_{X} G: A$, then $M, P \vdash_{X} G: B$, where $X$ is either NCK $+\mathrm{nmp}$ or NCK + nid + nmp.

This statement is needed to handle the contraction in case the counterfactual conditional formula is eliminated by the $n m p$ rule.

Theorem 6.6. The following propositions hold:

(1) Every derivation d in NCK + nmp and NCK + nid + nmp is normalizable.

(2) If $M \vdash_{X} G$; $A_{i}^{n}: B$, where the label $A_{i}^{n}$ does not occur in $M$, and there is a normal derivation of $A \leftrightarrow C$ in $X$, then $M \vdash_{X} G$; $C_{j}^{n}: B$, where $X$ is either $N C K+n m p$ or $N C K+$ nid $+n m p$.

(3) If $M \vdash_{X} G$; $A_{i}^{n}: B$, where the label $A_{i}^{n}$ does not occur in $M$, and $P \vdash_{X}$ $G: A$, then $M, P \vdash_{X} G: B$, where $X$ is either $N C K+n m p$ or $N C K+$ nid + nmp.

Proof. Let us call $C u t(c, h)$ the assertion that the property (i) holds for the cutrank $c$ and the sum $h$ of the heights of all critical cuts in $d$. Let us call $S u b(c)$ the assertion that (ii) holds for any $M$ and any labelled formula $H: D$ of complexity $c$; let us call $M P(c)$ the assertion that (iii) holds for any $M$ and $P$ and any labelled formula $H: D$ of complexity $c$. We show the following facts:

(1) $\forall c \forall h\left(\forall c^{\prime}<c C u t\left(c^{\prime}, h\right) \wedge \forall h^{\prime}<h C u t\left(c, h^{\prime}\right) \rightarrow C u t(c, h)\right)$

(2) $\forall c(\forall h C u t(c, h) \rightarrow S u b(c))$.

(3) $\forall c(\forall h C u t(c, h) \rightarrow M P(c))$. 
Let us first prove (1). The proof of (1) is the same as the proof of (i) except for the case where a labelled formula of the form $G: A>B$ is firstly introduced by the rule $>I$ and then eliminated by the rule $n m p$, i.e. except for the case where we have the following situation:

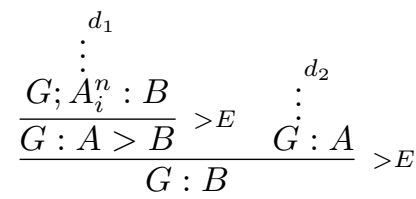

Since $|\mathrm{B}|<\mid A>B$ and the label $A^{n}$ does not occur in the premisses of $d_{1}$, because of the restriction of the rule $>I$, by inductive hypothesis we can apply (3) to the derivation $d_{1}$ and the derivation $d_{2}$ obtaining a derivation $d_{3}$ of $G: B$ (from the same premisses of $d_{1}$ and $d_{2}$ ). This is exactly what we were looking for.

Before passing to the proofs of (2) and (3), let us remind two standard properties of normal deductions which have been already mentioned before and will be useful in what follows.

Theorem 6.7. Let $d$ be a normal deduction in $N C K+n m p$ or $N C K+n i d+n m p$ and let $\beta=G_{1}: A_{1}, G_{2}: A_{2}$, . . , $G_{k}: A_{k}$ be a branch in $d$. Then there is a labelled formula occurrence $G_{i}: A_{i}$, called the minimum formula in $\beta$, which separates two (possibly empty) parts of $\beta$, called the E-part and the I-part of $\beta$, with the properties:

- Each $G_{j}: A_{j}$ in the E-part (i.e. $j<i$ ) is a major premise of an E-rule.

- $G_{i}: A_{i}$, provided that $i \neq k$, is premiss of an I-rule or of the rule $\neg E$.

- Each $G_{j}: A_{j}$ in the I-part, except the last one (i.e. $i<j<k$ ), is a premiss of an I-rule.

Proof. The proof can be developed in the same way as the proof of Theorem 3 in [18, p. 41-42].

Corollary 6.8. Let $d$ be a derivation of $G$ : A in $N C K+n m p$ or $N C K+n i d+n m p$ without undischarged assumptions, which is normal and such that $G: A$ is not atomic. Then the derivation $d$ ends with an I-rule.

Proof. The proof can be developed in the same way as the proof of Exercise $6.26 \mathrm{D}$ in $[22$, p. 148].

Let us now pass to (2). The proof of (2) is the same as the proof of (ii) except for the case where $G ; A_{i}^{n}: B$ has been introduced by the rule $n m p$. This case can easily be dealt with by applying the inductive hypothesis on the premises and then by reapplying the rule $n m p$. 
Let us finally prove (3). We proceed by induction on the height $h$ of the derivation of $G ; A_{i}^{n}: B$ from $M$. We distinguish cases according to the last rule that has introduced $G ; A_{i}^{n}: B$. If $G ; A_{i}^{n}: B$ is an assumption, $G: B$ is an assumption too. If $G ; A_{i}^{n}: B$ has been derived by a rule $\mathcal{R}$ that is either a propositional rule or one of the rules $>I$ and $n m p$, then we apply the inductive hypothesis and then the rule $\mathcal{R}$ again. If $G ; A_{i}^{n}: B$ has been derived by the rule $>E$, then we consider the following problematic case:

$$
\frac{d_{3}}{\vdots: \stackrel{d_{4}}{D}>B \quad D \stackrel{!}{\leftrightarrow} A}>\text { }>E
$$

To solve this case, we proceed in the following way. Let us consider the derivations $d_{4}$ of $D \leftrightarrow A$; this derivation is normal and without undischarged assumptions. Since $d_{4}$ is normal, does not contain any undischarged assumption and ends up with non-atomic formula, we can apply on it Corollary 6.8 and thus know that $d_{4}$ terminates with an introduction rule. Given the form of its last formula, namely $D \leftrightarrow A$, then we know that its last rule is the rule $\wedge I$. In particular, we know that the derivation $d_{4}$ ends with $\wedge I$ applied on $A \rightarrow D$ and $D \rightarrow A$.

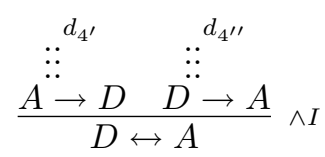

Let us now consider the derivation $d_{4^{\prime}} ; d_{4^{\prime}}$ is in normal form, does not contain undischarged assumptions and ends up with non-atomic formula. Therefore, even on $d_{4^{\prime}}$ we can apply Corollary 6.8 and thus know that it terminates with an introduction rule. Given the form of its last formula, namely $A \rightarrow D$, then $d_{4^{\prime}}$ have as its last rule the rule $\rightarrow I$; more precisely $d_{4^{\prime}}$ is nothing but the result of the application of the rule $\rightarrow I$ on a derivation $d^{\prime}$ from $A$ to $D$.

Let us now consider the derivation $d^{\prime}$. By applying several times Lemma 5.3 on it, we obtain a proof $d^{\prime} *$ from the labelled formula $G: A$ to the labelled formula $G: D$. Let us now combine together such a derivation $d^{*}$ with the derivation $d_{2}$ of $G: A$, obtaining a derivation $d^{*}$ of $G: D$ from the same premisses of $d_{2} . d^{*}$ might contain a cut of cutrank $|A|$. In this case, since $|A|<$ $|A>B|$, we can apply the inductive hypothesis on (1) and obtain a normal derivation of $G: D$. We use this normal derivation and the derivation $d_{3}$ of $G: D>B$ to apply the rule $n m p$ and obtain a derivation of $G: B$ as desired.

Finally, if $G ; A_{i}^{n}: B$ has been obtained by the rule nid, then we consider the derivation $d_{2}$ of $G: A$ and thanks to Lemma 5.4 we get the desired derivation. 


\section{From natural deduction calculi to sequent cal- culi and adequateness theorem}

In this section we show how to transform natural deduction calculi into sequent calculi. We exploit this result to prove the adequateness theorem of the NCK* calculi.

Theorem 7.1. Let $X \in N C K^{*}$ and $\mathbf{X} \in G C K^{*}$. For all $X$ and $\mathbf{X}$, for all multiset of formulas $M$ and all labelled formula $G: A$

$$
\text { if } M \vdash_{X} G: A \text {, then } \vdash M \Rightarrow G: A \text { in } \mathbf{X}
$$

Proof. By induction on the height of the derivations in X. We distinguish cases according to the last rule applied in the derivation $d$ of $X$. If $G: A$ is an assumption, then is translated as the axiom $G: A \Rightarrow G: A$. If $G: A$ has been derived by means of a propositional introduction or elimination rule, then we follow the standard strategy, see [22, p.190-191]. If $G: A$ is of the form $G: B>C$ has been derived by means of the rule $>I$, then we have an object of the following form

$$
\begin{gathered}
M \\
\vdots \\
\frac{G ; B_{i}^{n}: C}{G: B>C}>I
\end{gathered}
$$

where the label $B_{i}^{n}$ does not occur in $M$. By the inductive hypothesis, we have that the sequent $M \Rightarrow G ; B_{i}^{n}: C$ is derivable in $\mathbf{X}$. By applying the rule $>R$, we obtain $M \Rightarrow G: B>C$.

If $G: A$ is of the form $G ; C_{j}^{n}: B$ has been derived by means of the rule $>E$, then we have an object of the following form:

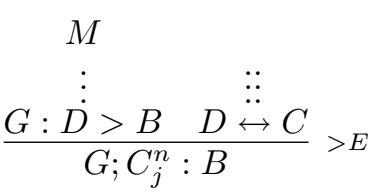

By the inductive hypothesis we have that the sequents $M \Rightarrow G: D>B$ and $\Rightarrow D \leftrightarrow C$ are derivable in $\mathbf{X}$. We consider the derived sequent $G ; C_{j}^{n}: B \Rightarrow$ $G ; C_{j}^{n}: B$. By applying on this sequent and the sequent $\Rightarrow D \leftrightarrow C$ the rule $>L$, we obtain $G: D>B \Rightarrow G ; C_{j}^{n}: B$. By applying a cut on $M \Rightarrow G: D>B$ and $G: D>B \Rightarrow G ; C_{j}^{n}: B$, we obtain $M \Rightarrow G ; C_{j}^{n}: B$, which is the desired conclusion.

If $G: A$ is of the form $G ; B_{i}^{n}: B$ and has been derived by means of the rule nid, then we have to show that the sequent $\Rightarrow G ; B_{i}^{n}: B$ is derivable in $\mathbf{X}$. This can be easily done by considering axioms of the form $G ; B_{i}^{n}: B \Rightarrow G ; B_{i}^{n}: B$ and by applying on them the rule $i d$.

If $G: A$ has been derived by means of the rule $n m p$, then we have an object of the following form 


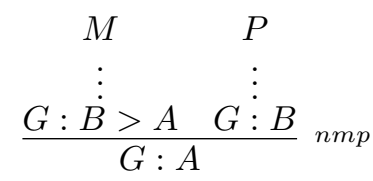

By the inductive hypothesis, we have that the sequent $M \Rightarrow G: B>A$ and the sequent $P \Rightarrow G: B$ are both derivable in $\mathbf{X}$. We consider axioms of the form $G: B>A, G: B \Rightarrow G: B$ and of the form $G: B>A, G: A \Rightarrow G: A$ and we apply on them the rule $m p$, obtaining the sequent $G: B>A, G: B \Rightarrow G: A$. We first apply a cut on the sequent $M \Rightarrow G: B>A$ and the sequent $G: B>$ $A, G: B \Rightarrow G: A$, getting $G: B, M \Rightarrow G: A$. Then we apply a second cut on $P \Rightarrow G: B$ and $G: B, M \Rightarrow G: A$, thus obtaining the desired conclusion $M, P \Rightarrow G: A$.

We now show soundness and completeness of the calculi NCK* with respect to the appropriate semantics. In order to establish this result we use Theorem 7.1 .

\section{Theorem 7.2.}

(i) If $G: A$ is derivable from a multiset $M$ in a calculus $X \in N C K^{*}$, then $(G: A)^{\tau}$ is a logical consequence of the multiset $(M)^{\tau}$ in the corresponding class of frames $\mathfrak{C}+X, X \in\{\mathfrak{i} \mathfrak{i}, \mathfrak{m} \mathfrak{p}\}$.

(ii) If $A$ is valid in a class of frames $\mathfrak{C}+X, X \in\{\mathfrak{i d}, \mathfrak{m} \mathfrak{p}\}$, then $A$ is derivable in the corresponding natural deduction calculus $X \in N C K^{*}$.

Proof. (i) follows from Theorem 4.1 and Theorem 7.1. In order to further acquaint the reader with the calculi $\mathrm{NCK}^{*}$ we verify (ii). We present the deductions of the axioms ID and MP, and we show that the rules RCK and RCEA are derivable in the calculi NCK*. As for the classical axioms and the rule of modus ponens, the deductions are the standard ones. ${ }^{7}$

RCK rule

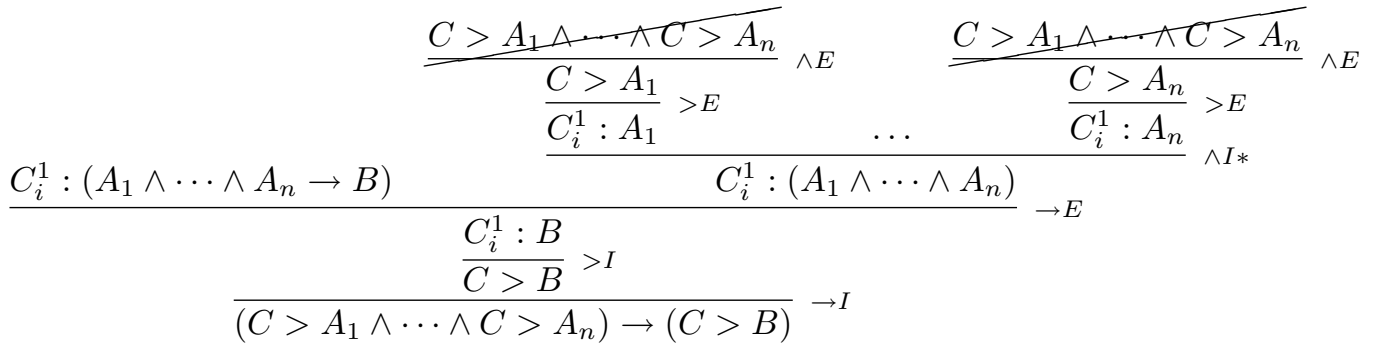

\footnotetext{
${ }^{7}$ We will use $\mathcal{R}^{*}$ to denote several applications in a row of the rule $\mathcal{R}$.
} 
where $C_{i}^{1}:\left(A_{1} \wedge \cdots \wedge A_{n} \rightarrow B\right)$ has been obtained from $\left(A_{1} \wedge \cdots \wedge A_{n} \rightarrow B\right)$ by Lemma 5.3 .

RCEA rule

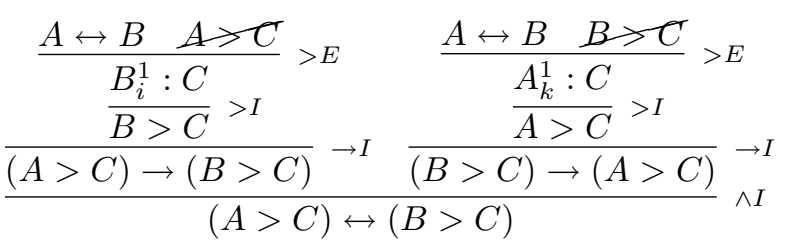

ID axiom

$$
{\overline{A_{i}^{1}: A}}^{\text {nid }}
$$

MP axiom

$$
\frac{\frac{A \gg B \quad A}{B}{ }_{n m p}}{\frac{A \rightarrow B}{(A>B) \rightarrow(A \rightarrow B)}} \rightarrow I
$$

\section{Conclusions}

In this paper we have focussed on the proof theory for counterfactual logics based on Nute semantics. In particular we have introduced labelled sequent calculi and labelled natural deduction calculi for the systems CK*. We have proved that these calculi satisfy many important properties. In the light of these results, we see two main lines of future research. On the one hand, we would like to extend our labeling method to other logics based on Nute's semantics, like those characterized by the axioms CEM and CSO, see [12]; on the other hand, we believe it would interesting to draw a formal comparison between our calculi and the other calculi, introduced in [1, 14], for the logics based on Nute semantics.

In the last decade research in proof theory for non-classical logics has known great developments; nevertheless, the interesting question of finding analogous sequent calculi and natural deduction calculi for the same (non-classical) logic has been left aside. We hope that this paper can be seen as a first step towards filling this gap. 


\section{References}

[1] R. Alenda, N. Olivetti, and G. L. Pozzato. Nested sequent calculi for normal conditional logics. Journal of Logic and Computation, 8:1-48, 2014.

[2] A. Artosi, G. Governatori, and A Rotolo. Labelled tableaux for nonmonotonic reasoning: Cumulative consequence relations. Journal of Logic and Computation, 12:1027-1060, 2002.

[3] K. Brünnler. Deep sequent systems for modal logic. In G. Governatori, I. Hodkinson, and Y. Venema, editors, Advances in Modal Logic, Volume 6, pages 107-119. College Publications, London, 2006.

[4] F. B. Chellas. Conditional logics. Journal of Philosophical Logic, 4:133-153, 1975.

[5] G. Crocco and P. Lamarre. On the connection between non-monotonic inference systems and conditional logics. In B. Nebel and E. Sandewall, editors, Principles of Knowledge Representation and Reasoning: Proceedings of the 3rd International Conference KRÕ92, pages 565-571. 1992.

[6] H. C. M. de Swart. A gentzen- or beth- type system, a practical decision procedure and a constructive completeness proof for the counterfactual logics VC and VCS. Journal of Symbolic Logic, 48:1-20, 1983.

[7] B. Hill. Fiction, counterfactuals: the challenge for logic. In S. Rahman and O. Pombo, editors, Unity of Science. Vol. II: Special Sciences, pages 277-299. Springer, 2012.

[8] C. Hitchcock. The intransitivity of causation revealed in equations and graphs. Journal of Philosophy, 98:273-299., 2001.

[9] A. Indrzejczak. Natural Deduction, Hybrid Systems and Modal Logic. Springer, Dordrecht, 2010.

[10] D. Lewis. Counterfactuals. Blackwell, Basil, 1973.

[11] D. Nute and C. B. Cross. Conditional logic. In D. Gabbay and F. Guenthner, editors, Handbook of philosophical logic, Volume 4, pages 1-99. Kluwer Academic Publisher, 2002.

[12] J.J. Nute. Topics in Conditional Logics. Reidel, Dordrecht, 1980.

[13] N. Olivetti and G. L. Pozzato. A standard internal calculus for lewis' counterfactual logics. Tableaux 2015, pages 270-286, 2015.

[14] N. Olivetti, G. L. Pozzato, and C.B. Schwind. A sequent calculus and a theorem prover for standard conditional logics. ACM Transactions on Computational Logic, 8:40-87, 2007. 
[15] D. Pattinson and L. Schroder. Generic modal cut elimination applied to conditional logics. Logical Methods in Computer Science, 7:1-28, 2011.

[16] F. Poggiolesi. The method of tree-hypersequents for modal propositional logic. In D. Makinson, J. Malinowski, and H. Wansing, editors, Trends in Logic: Towards Mathematical Philsophy, pages 31-51. Springer, 2009.

[17] F. Poggiolesi. Gentzen Calculi for Modal Propositional Logics. Springer, Dordrecht, 2010.

[18] D. Prawitz. Natural Deduction, A proof theoretical study. Almqvist and Wiskell, Uppsala, 1965.

[19] G. Restall. Proofnets for S5: sequents and circuits for modal logic. In C. Dimitracopoulos, L. Newelski, and D. Normann, editors, Logic Colloquium 2005, pages 151-172. Cambridge University Press, Cambridge, 2007.

[20] R. Stalnaker. A theory of conditionals. In N. Rescher, editor, Studies in Logical Theory, pages 98-112. Blackwell, Oxford, 1968.

[21] R. H. Thomason. A fitch-style formulation of conditional logic. Logique et Analyse, 13:397-412., 1970.

[22] A. Troelestra and H. Schwichtenberg. Basic Proof Theory. Cambridge University Press, Cambridge, 1996. 